\title{
Kernos
}

Revue internationale et pluridisciplinaire de religion

grecque antique

13 | 2000

Varia

\section{Françoise MIEs (éd.), Toute la sagesse du monde. Hommage à Maurice Gilbert, s.j. Pour le 65e anniversaire de l'exégète et du recteur}

\section{André Motte}

\section{(2) OpenEdition}

Journals

Édition électronique

URL : http://journals.openedition.org/kernos/1338

DOI : 10.4000/kernos. 1338

ISSN : 2034-7871

\section{Éditeur}

Centre international d'étude de la religion grecque antique

\section{Édition imprimée}

Date de publication : 1 janvier 2000

ISSN : 0776-3824

\section{Référence électronique}

André Motte, «Françoise mies (éd.), Toute la sagesse du monde. Hommage à Maurice Gilbert, s.j. Pour le 65e anniversaire de l'exégète et du recteur », Kernos [En ligne], 13 | 2000, mis en ligne le 16 juin 2011, consulté le 22 septembre 2020. URL : http://journals.openedition.org/kernos/1338 ; DOI : https:// doi.org/10.4000/kernos.1338 
démonstratif que documentaire, mais l'historien rencontre ici des difficultés spécifiques. Après une introduction méthodologique appropriée, l'A. aborde avec maîtrise une série de problèmes délicats qui vont du recensement ( un événement reconstruit 》), censé avoir commandé le voyage de Marie et Joseph, au procès de Jésus (celui de Socrate apparaît quelquefois en filigrane dans les témoignages) et à la crucifixion en passant notamment par le thème des généalogies, de la naissance miraculeuse, de la thaumaturgie et du miracle. Abordant des questions non moins délicates, le chapitre consacré à l'Église de la Pentecôte nous vaut d'intéressantes considérations sur l'inspiration et la communication, l'occasion de faire apparaître à la fois des ressemblances et des différences entre les sensibilités grecque et chrétienne, à propos, par exemple, des visions en songe, des apparitions et des phénomènes d'extase.

Les sections suivantes, après un arrêt sur l'Église de Jérusalem, ne sont pas moins passionnantes et riches de perspectives nouvelles. Pour faire bref, disons seulement qu'elles s'attachent à l'examen de problèmes liés à la pénétration et à la diffusion du christianisme dans le monde grec, avec une attention spéciảle accordée aux difficultés d'intégration et aux crises identitaires (e.g. : l'apparition de l'hérésie, la question des «faux prophètes », la magie, le culte impérial) ainsi qu'aux rapports entre les chrétiens et le pouvoir (en particulier, la difficile question des persécutions et de leur fondement juridique).

L'ouvrage se lit d'un bout à l'autre avec un intérêt soutenu. À défaut de la compétence nẹcessaire pour juger de certaines positions de fond, on ne peut manquer d'apprécier l'esprit critique et la rigueur scientifique qui président à toutes les enquêtes. L'ouvrage s'adressant à un large public cultivé, on comprend que l'appareil justificatif se limite le plus souvent, dans les notes, à de simples références aux sources anciennes, mais il convient d'attirer l'attention sur la très riche bibliographie commentée qui figure à la fin de l'ouvrage (p. 405-451) et qui suit l'ordre des onze chapitres. S'y ajoutent un relevé des sources, un index des noms de lieux et un autre des noms de personnes, une table des neuf cartes, fort bienvenues, disséminées dans le livre ainsi qu'une table des matières détaillée. La présentation, on le devine, est à tous égards très soignée et elle contribue, avec le style sobre et clair, à l'agrément de la lecture.

André Motte

(Université de Liège)

Françoise Mies (éd.), Toute la sagesse du monde. Hommage à Maurice Gilbert, s.j. Pour le $65^{e}$ anniversaire de l'exégète et du recteur, Namur, Presses universitaires, 1999.1 vol. $14,5 \times 20,5 \mathrm{~cm}, 768$ p. (Connaître et croire, 4). ISBN : 2-87037-278-7.

Le R.P. M. Gilbert vient de quitter la fonction de recteur des Facultés universitaires Notre-Dame de la Paix de Namur qu'il exerçait depuis 1993, après avoir été auparavant recteur des Instituts Bibliques de Rome (1978-1984) et de Jérusalem (1984-1992), deux institutions dans lesquelles il a repris actuellement son enseignement. Sa très copieuse bibliographie, que détaille le volume (p. 705-731), témoigne non seulement d'une compétence particulièrement avérée dans le domaine biblique, mais aussi d'une fine connaissance de l'hellénisme. Méritent notamment d'être épinglés à cet égard sa thèse de doctorat : La critique des dieux dans le Livre de la Sagesse, Rome, 1973, ainsi qu'un de ses tout derniers articles: La sagesse de Salomon et l'bellénisme, dans Hieros. Bulletin de la Société belgoluxembourgeoise d'bistoire des religions, 4 (1999), p. 11-31. Les livres sapientiaux ayant été son sujet de prédilection (à noter, en particulier, cet important ouvrage collectif dont il est l'éditeur scientifique : La sagesse de l'Ancien Testament, 
Leuven, Peeters, $1990^{2}$ ), on ne s'étonne pas du thème choisi pour lui rendre hommage.

Sous ce bel intitulé sont rassemblées trente-quatre contributions des plus variées qui illustrent fort bien l'universalité et la richesse du thème, tant dans le temps que dans l'espace. Poussant l'investigation jusque dans la préhistoire (J. Ries, L'art pariétal, livre de sagesse de la prébistoire ?), la recherche fait une place de choix, on n'en sera pas surpris, aux œuvres bibliques de l'Ancien comme du Nouveau Testament, avec des études, en forme parfois de méditation, sur le livre de Job (J. Lévêque, F. Mies), sur le jugement de Salomon (J.-N. Aletti) et l'histoire idéalisée de ce roi (P. Gibert), sur le Cantique des Cantiques (X. Dijon), sur le Psaume 9 (B. Costacurta, «L'bomme est comme l'berbe »), sur la conception et la gestion du temps dans le judaïsme (A. Guigui), etc., et aussi sur Luc (J. Radermakers), Jean (L. Devillers) et Paul (P. Garuti, Sophia logou, Sopbia theou). Mais le thème trouve pareillement de nombreux prolongements dans l'actualité, à commencer par celle de la réflexion philosophique. Y a-t-il une sagesse des modernes?, se demande É. Ganty tandis qu'il scrute la pensée de Luc Ferry et d'A. Comte-Sponville. Ce sont ces deux mêmes penseurs, auxquels se joint cette fois $M$. Blondel, qui alimentent la réflexion du philosophe P. Favraux tandis que J. Baufay, récemment décédé, évoque La sagesse boiteuse de Kierkegaard. Plusieurs problèmes contemporains de société trouvent aussi leur place, de façon parfois inattendue : sagesse et droits de l'homme (J.B. Nagy), sagesse et bioéthique, à la lumière notamment des interpellations de Levinas, de Jonas et de Ricœur (J. Duchêne), sagesse et approche psycho-sociale du handicap et de la pauvreté (M. Mercier), l'humanité du dément vu à la lumière des sagesses hébrai que et grecque (N. Rigaux).

Pas plus que l'Antiquité, l'Occident et le Proche-Orient ne bénéficient ici d'un monopole. J. Scheuer examine La marche à la sagesse selon le bouddhisme, cependant que $\mathrm{C}$. Jeffré fait voir la sagesse comme un possible lieu de rencontre entre christianisme et religion chinoise. Plus largement encore, c'est à la sagesse des nations qu'en appelle A. Chouraki, pressentant « un monde nouveau en train de naitre sous nos yeux » et qui « exige de nous tous et de chacun un sacrifice d'identité ".

La Grèce antique, on s'en doute, n'est pas absente de ce vaste concert. Elle apparaît tantôt en filigrane quand est évoquée l'inspiration épicurienne d'un Comte-Sponville ou que sont opposées sagesse des Anciens et sagesse des Modernes, tantôt aussi en pleine lumière lorsque J. Ladrière, par exemple, prend pour point de départ de sa réflexion sur la science contemporaine la conception aristotélicienne de la sopbia, revisitée par saint Thomas, ou que L. Isebaert, attentif pour sa part à la dimension religieuse de la sagesse grecque, en étudie les origines chez Hésiode; son bel article montre en quoi l'œuvre du poète béotien s'apparente à la réflexion des premiers philosophes de l'Ionie ainsi qu'à la tradition des sept sages.

La succession des articles ne suit pas un ordre chronologique, mais thématique, ce qui concourt à faire apparaître les principales articulations de cette notion de sagesse qui met en cause les rapports entre l'homme et Dieu, l'individu et la société, la vie et la mort, l'intelligence et l'amour, les mots et l'au-delà des mots. C'est dans cette dernière thématique qu'est notamment classée l'étude de JM. André sur Les livres de Sagesse dans l'ouvre d'Olivier Messiaen, un petit air de musique auquel fait écho l'intitulé de la dernière contribution, due au dédicataire même de l'ouvrage: "N'empêche pas la musique ». Il s'agit d'un conseil que dispense le livre de Ben Sira à celui que les participants au banquet ont choisi comme président. C'est qu'en plus du bon vin et des discours, il sied, 
explique-t-il, de faire place aussi à la musique, dont la vertu contribue à parfaire le symposion. Appliquant le propos à la charge qu'il vient de quitter, le recteur honoraire l'estime particulièrement de mise pour qui préside à un débat d'idées. Mais du conseil à la pratique, ajoute-t-il, " il y a plus qu'un pas... ".

La sagesse, dirait-on, prend ici un nom propre, parmi bien d'autres à découvrir dans ce beau volume d'hommage dont on apprécie aussi la présentation soignée et qui, outre la bibliographie du P. M. Gilbert, comporte encore une brève présentation de chaque auteur, un index des références bibliques ainsi qu'une tabula gratulatoria, réservée aux membres des Facultés de Namur.

André Motte

(Université de Liège)

Jacqueline Fabre-Serris, Mythologie et littérature à Rome. La réécriture des mythes aux $j^{\text {ers }}$ siècles avant et après J.-C., Éditions Payot Lausanne, Sċiences humaines, 1998.1 vol. $14 \times 22,5 \mathrm{~cm}, 271$ p. ISBN : 2-601-03228-6.

On a dit que les Romains n'avaient pas de mythes, seulement des légendes. Si l'on en croit le De natura deomm de Cicéron, c'est là la distinction fondamentale qui différencie religion grecque et religion romaine. Les récits mythologiques sur les dieux seraient le propre des Grecs, tandis que les Romains se font de la religion une idée plus pratique. Rituel, prise des auspices, prophéties, telles en sont les dimensions principales. Aux yeux des Romains, les récits sur les dieux n'avaient aucune importance. C'est sans doute pour cette raison que des historiens "hypercritiques" comme Th. Mommsen, G. Wissowa ou E. Pais écrivaient l'histoire de Rome en s'en tenant aux événements historiquement établis. Il a fallu attendre des savants tels que J. Bayet, G. Dumézil ou J. Hubaux pour prendre conscience d'une autre dimension de la pensée romaine : celle des récits sur les origines et le développement de la cité. C'est alors que l'on redécouvre l'importance du premier livre de Tite-Live et des mythes qu'il contient ${ }^{1}$. Il faut attendre une période relativement tardive dans l'histoire romaine, le $\mathrm{I}^{\text {er }}$ siècle avant J.-C., pour voir la mythologie occuper une place importante à Rome. À cette époque, Rome est déjà une société élaborée, dont la culture, la littérature et la pensée ont été imprégnées par une influence grecque importante. Les auteurs latins qui ont conservé les mythes italo-romains ou grecs, introduits très tôt en Italie, les « réélaborent » et les remodèlent à leur guise en y ajoutant des éléments nouveaux. Ils projettent dans la mythologie la crise des consciences et des valeurs traditionnelles que traverse l'Urbs. Ainsi la mythologie sert de véhicule à un message moral et politique d'une société en crise. C'est en se plaçant de ce point de vue que l'auteur tente de montrer, en prenant des exemples précis, de la fin de la République au temps de Néron, comment les poètes ont métamorphosé les mythes et ont centré leur réflexion sur des thèmes précis : les origines de la cité, la légitimité du pouvoir, les valeurs civiques, les passions et la nature de l'homme.

L'introduction évoque d'abord les sources : textes historiques de la fin de la République et du début de l'Empire (Cicéron, Tite-Live, Denys d'Halicarnasse), œuvres poétiques, d'Ennius à Virgile, Properce et Ovide, écrits relatifs à la grammaire, à la religion, au droit ou à la philosophie. Elle traite ensuite des

1 B. Llou-Gilne, Une lecture "religieuse" de Tite-Live I. Cultes, rites de la Rome archaique, Paris, 1998 (Études et commentaires, 105). 